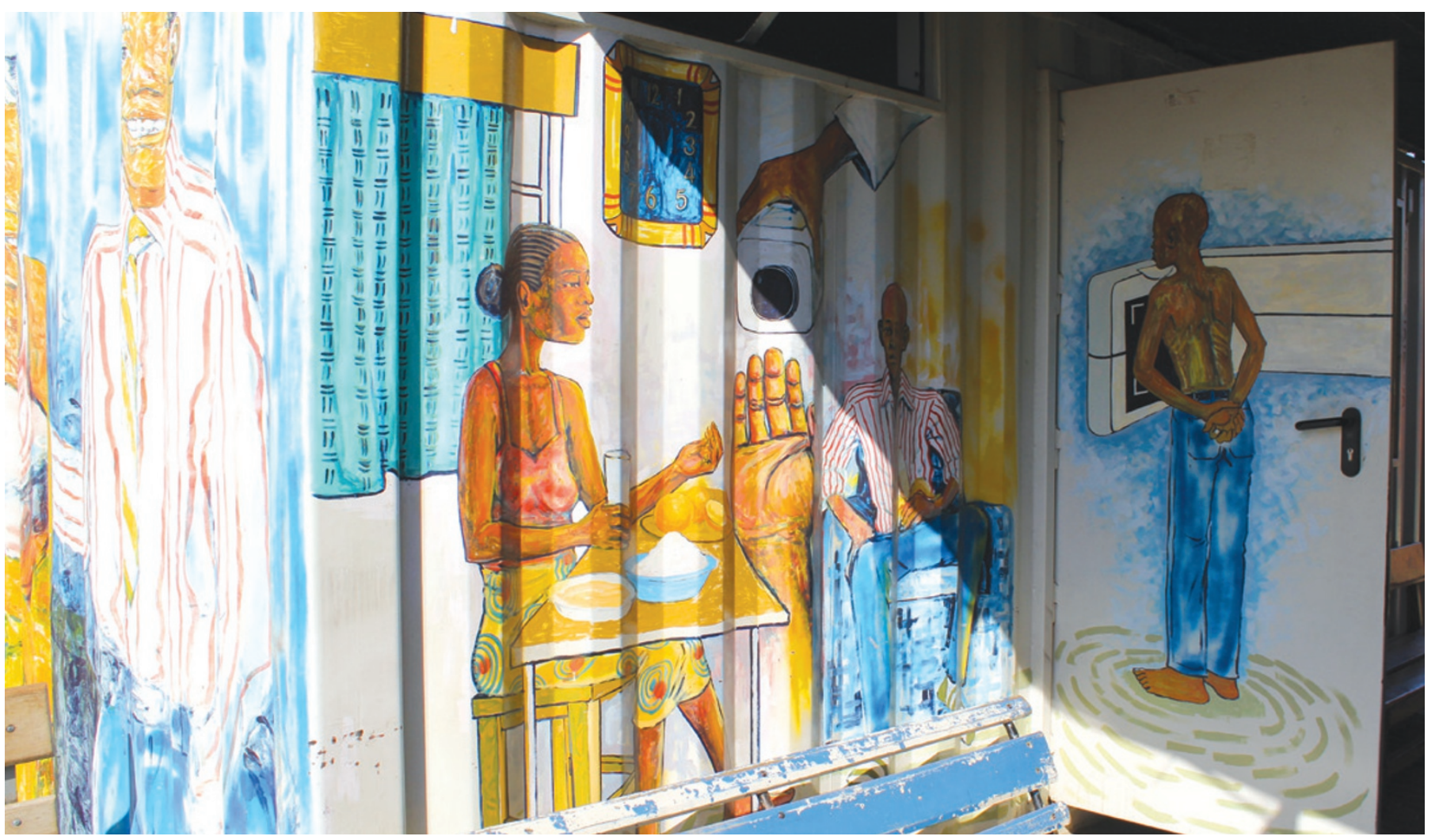

The health clinic in Kanyama, a slum district of Lusaka, Zambia, is trying out a new device to test for tuberculosis.

DIAGNOSIS

\title{
Waiting for results
}

\section{There are several new tests for tuberculosis in the pipeline, but they must be shown to be effective in areas with limited resources and a heavy burden of HIV.}

\section{BY CATHERINE DE LANGE}

I obias Hamooya says he is feeling a bit better these days, but even to the casual observer it is clear that he is still not doing well. Sitting on the porch outside the Macha Mission Hospital in rural Zambia, his slow, staggered speech is punctuated by a violent cough, and just talking seems to leave him drained.

A few weeks ago Hamooya's cough got so bad he left his wife to look after their newborn baby and six other children and travelled the 150 kilometres to get here. Did he have any idea what was wrong with him? His answer needs no translation: "TB". Since arriving at the clinic they also tested him for HIV, and the results came back positive for that too. Once his tuberculosis (TB) medication kicks in, he will begin taking antiretrovirals (ARVs).

Pretty much everyone in the region is infected with TB, says John Spurrier, medical adviser at the hospital where Hamooya is being treated. "In most people your body handles it. You may go all your life and never have a problem. Now with HIV, which destroys the immune system, it becomes a big issue."

The highest proportion of new TB cases is in sub-Saharan Africa: more than 260 people per 100,000 in 2011. By comparison, in the same year France saw 4 cases per 100,000 . And the region is in the grips of an HIV epidemic; TB kills more people living with HIV than anything else, and detection and treatment of TB is vastly complicated by HIV co-infection. Where once treatment programmes operated independently, many countries like Zambia now try to test and treat the two together. In 2004, just 3\% of TB patients in the World Health Organization (WHO)'s African Region were tested for $\mathrm{HIV}$; in 2011, it was 69\%. Hamooya was one of the lucky ones: detecting TB is extremely difficult in patients who also have an HIV infection.

HIV has an effective point-of-care test that can quickly and accurately detect infection and is practical and cheap enough for use in rural clinics. TB does not. In recent years, finding such a test for $\mathrm{TB}$ has been a major public-health goal, and these efforts are beginning to bear fruit. Over the next few months, a new test called Xpert MTB/RIF that detects TB DNA will enter clinics in several high-burden countries in southern Africa; other tests are close behind. The question is, in the uncompromising settings that are home to the greatest burden of disease, will these new tests fulfil their potential?

\section{NEED FOR SPEED}

A fast TB diagnosis is important. The sooner a patient is diagnosed, the sooner they can start treatment to mitigate the debilitating symptoms of TB and limit the potential for transmission. But there is an added imperative for HIV-positive patients. "Starting someone on ARVs who has [untreated] TB means that, as their immune system recovers, they can get very sick," says Spurrier.

Spurrier is referring to IRIS - immune reconstitution inflammatory $\checkmark$ NATURE.COM For more on the simple fluorescent dye test for TB, visit go.nature.com/wmtneq 
syndrome. "Most of the symptoms you have from disease are [from] your body fighting the disease, not from the virus itself. So if your immune system is not working, you can have disease all through your body and have relatively few symptoms," he says. Once the rebuilt immune system tries to fight $\mathrm{TB}$, the immune response can be lethal.

The standard method for testing for TB in the clinic, smear microscopy, is not very fast. The technique has changed little since it was developed by German scientist Robert Koch in the 1880s; it involves staining and examining a sample of sputum - the mucus patients cough up - under a microscope, counting the bacteria and grading the severity of the infection. Although simple, this technique can take as long as a week to get a result and heavily depends on the experience of the technician performing the test. But even then it might be inconclusive: the test is estimated to detect TB in only around $40-50 \%$ of people with $\mathrm{HIV}^{1}$, rendering it impractical for parts of the world without the capacity for further tests. "Sputum is not very accurate: we miss people and then need to do additional work using $\mathrm{X}$-rays and so on," says Nzali Kancheya, TB programme director at the Center for Infectious Disease Research in Zambia (CIDRZ) in Lusaka.

\section{HIGH HOPES}

Xpert MTB/RIF aims to address these shortfalls, and its roll out is causing a big buzz. It is a fully automated machine and cartridge system that uses polymer chain reaction (PCR) to amplify and detect DNA sequences specific to Mycobacterium tuberculosis in sputum. The technology uses the GeneXpert system, an existing molecular testing device developed by molecular diagnostics company Cepheid, based in Sunnyvale, California. Cepheid, together with the Foundation for Innovative New Diagnostics in Geneva, Switzerland, and researchers from the University of Medicine and Dentistry of New Jersey, with additional funding from the National Institutes of Health in Bethesda, Maryland, developed the TB-specific system in 2008.

"It's made to be used by people with minimal training, even though it's a high-tech piece of equipment," says Monde Muyoyeta, one of the lead researchers on the Zambia AIDS Related Tuberculosis (ZAMBART) Project, a non-governmental organization formed through a collaboration between the University of Zambia's School of Medicine in Lusaka and the London School of Hygiene and Tropical Medicine. Sputum samples are loaded onto cartridges and inserted into the machine, which is connected to a computer to read off the results. In theory, this set-up should make it easy to diagnose patients, as the machine does all the analysis.

In December 2010, WHO recommended the use of Xpert MTB/RIF to diagnose TB and is monitoring a massive global roll out. Part of this comes in the form of the TB Xpert Project,

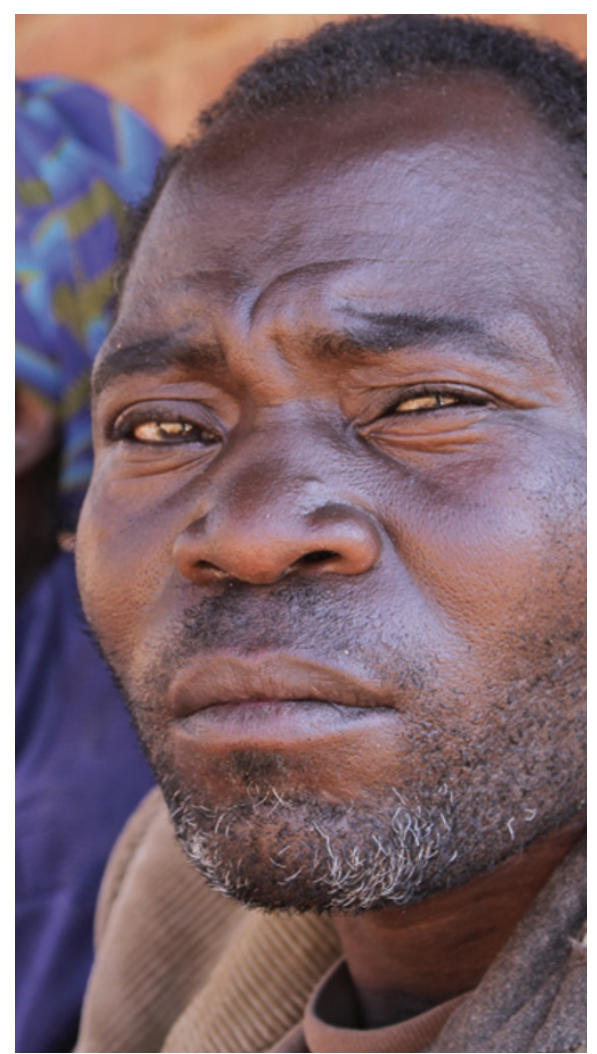

Tuberculosis patient Tobias Hamooya.

a US $\$ 25.9$ million programme funded by UNITAID, which is hosted by WHO in Geneva, and will provide over 220 machines and 1.4 million cartridges to 21 countries, mainly in East Africa and South-East Asia. Many countries are able to buy the system at a discount; as of March 2013, more than 6,100 machines and 2.3 million cartridges had been purchased $^{2}$ (see 'Xpert sales', page S12).

In Zambia, several Xpert machines have been bought for use in clinics, in anticipation of government guidelines due later this year. In the meantime, other machines are already in use for research, for instance by ZAMBART, to

\section{"Sputum is not} very accurate: we miss people and then need. to do additional work."

look at how effective it will be in resourcepoor settings. A lot rests on this technology, says Kancheya. "In Western countries they have a lot of other technologies, but in places like Zambia, GeneXpert will really help us," she says. But first, solid evidence about its utility needs to be collected from the field, with the ZAMBART Project taking the lead.

\section{PRACTICAL EXAM}

The health clinic in Kanyama is a bright spot in this densely populated slum in the west of Zambia's capital, Lusaka. It is also one of two sites chosen by ZAMBART to test GeneXpert in a primary healthcare setting. In addition to the GeneXpert units, the clinic is home to a make-shift chest X-ray clinic, housed away from the dust in a shipping container painted with scenes of TB care in Africa. Kanyama sees the highest rates of TB in the city, and the clinic is the ideal arena to study how the GeneXpert technology performs far from the sterile lab.

For example, GeneXpert depends on a continuous supply of electricity. "If you don't have that, how does it affect the performance of the tests?" says Muyoyeta. Regular power cuts are a reality in Kanyama. "With smear microscopy, if you run out of electricity you can just come back and finish your test later," she says. "But with GeneXpert, if you have a power outage and you don't have backup, you have to start all over again." If you are lucky, you have some leftover sample; if not, you will have to call the patient back to provide another. These issues are crucial in determining whether the system can perform as hoped. "If you're wasting samples and not getting the result the same day then it's not a point-of-care test," says Barry Kosloff, lab manager on the ZAMBART Project.

Then there is the cost. Under concessional pricing, the GeneXpert machine plus a computer to display the results costs around US $\$ 17,000$. The cartridges cost just under $\$ 10$ each. You need one cartridge per test, and the simplest version of the machine can process four cartridges at a time.

The cost is significant, Muyoyeta says. By comparison, "a microscopy slide is less than one dollar." And, when you add in the capital cost and additional costs like electricity, GeneXpert will be far more expensive. Technology like this might leave low- or middle-income countries more dependent on outside help. "I think it can make a difference," says Muyoyeta, "but it's so expensive there's no way that the minister of health can afford to roll out GeneXpert without support."

GeneXpert delivers results in as little as two hours, which means patients should be able to wait to receive them. In a busy month, Kanyama clinic will see as many as 500 patients for TB screening. At present, it takes staff between 24 and 48 hours to get results, which means a return trip to the clinic for patients. "Transportation is very difficult," says Kosloff. "If they are told they need to come back tomorrow or next week, they may say, 'Well, I need to decide - is my family going to eat tomorrow, or do I make another trip to the clinic?' If you can't get the results to people the same day, why put this device in such a difficult location?"

There are alternatives to Xpert that are less high-tech. One innovation is an improved form of smear microscopy that uses light-emitting diode (LED) bulbs and fluorescent dyes to show up the bacteria more clearly under the microscope. Although this technique picks up more M. tuberculosis than standard microscopy, it also mis-identifies contaminants, leading to false-positive results.

A different approach is to try to rule out, rather than rule in, TB in HIV-infected people 


\section{XPERT SALES}

Sales of GeneXpert devices, which can help to diagnose tuberculosis in HIV-infected people, have shot up since it was recommended by WHO in 2010.

The African region has the highest burden of HIV/TB coinfection and accounts for approximately $79 \%$ of HIV-infected tuberculosis cases.

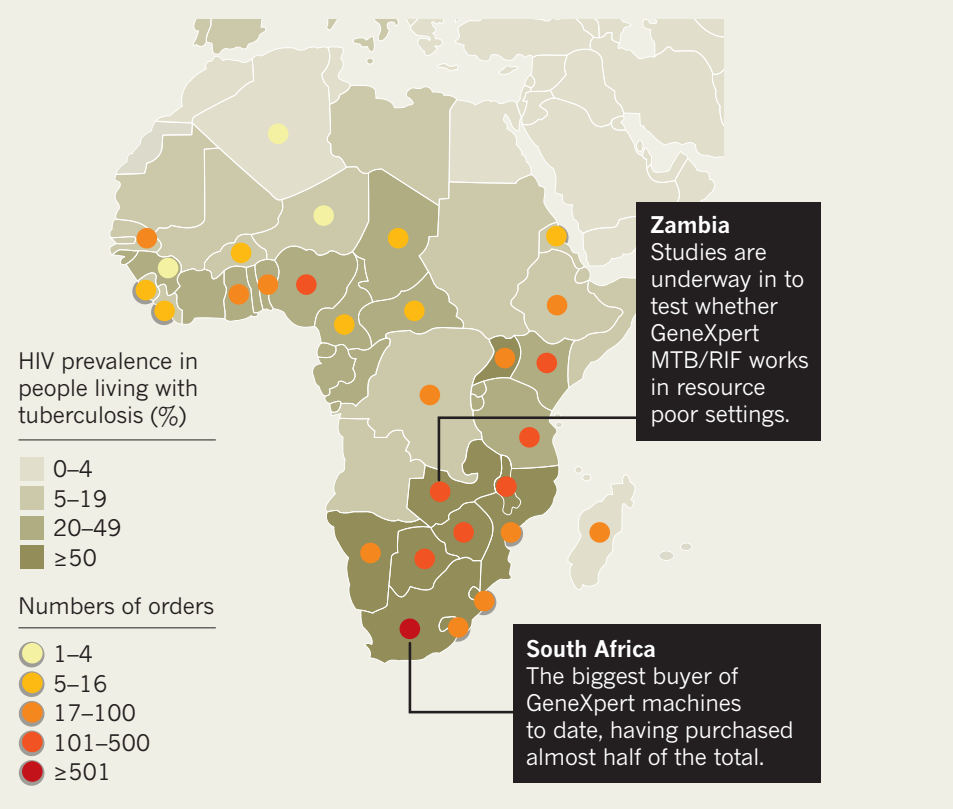

Cumulative number of GeneXpert instrument models and Xpert MTB/RIFcartridges procured.

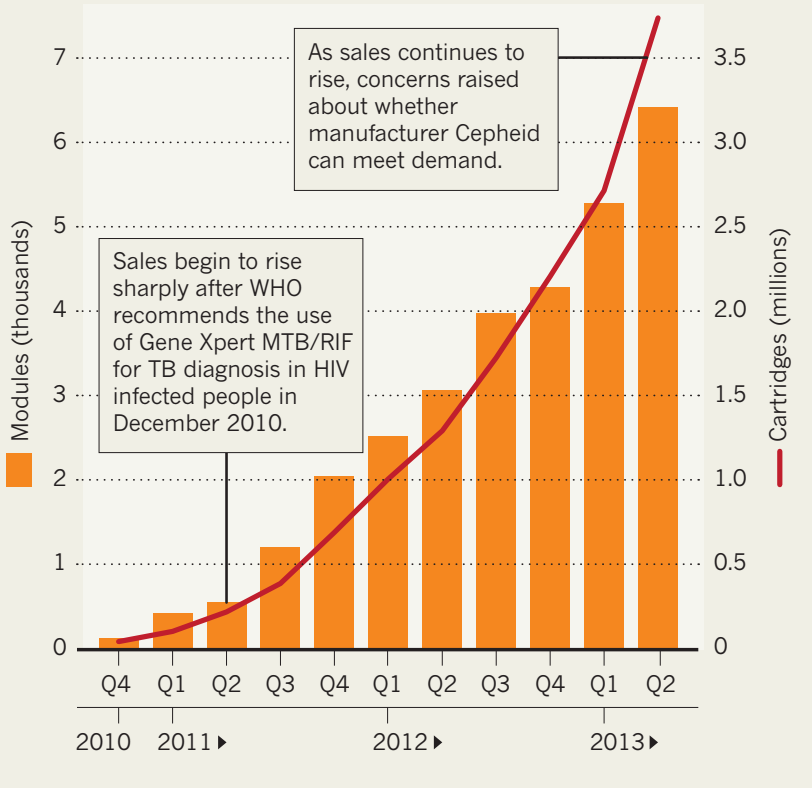

with suspected infection. Medical diagnostic firm Alere, based in Stockport, UK, has developed a dipstick that tests urine for lipoarabinomannan (LAM), a component of the cell wall of M. tuberculosis. Alere's test costs US $\$ 3.50$ per dipstick and provides results in 25 minutes. It works best in people with a heavily compromised immune system, such as HIV patients with advanced infection. Overall, sensitivity - the proportion of TB it finds - in people also living with HIV is moderate at $40-60 \%$, but its high specificity means that it can rule out TB in almost all uninfected people ${ }^{3}$.

Keertan Dheda, a pulmonologist at the University of Cape Town in South Africa, has conducted numerous studies into TB diagnostics. Dheda thinks that, despite its limitations, the dipstick is a valuable tool particularly given the difficulties of detecting TB in HIV-infected people. "In this category of patients, a urine-orientated approach may be very useful." Chris Smit, director of Alere's infectious disease unit, says he suspects a number of countries are waiting for WHO prequalification of the device before they begin to use it more widely. Alere is working with WHO to get approval by the end of 2014 .

\section{BIGGERISSUES}

The GeneXpert system has an additional benefit that these other tests do not: it also checks for resistance to rifampicin, one of the cocktail of four first-line drugs used to treat TB. And if a patient's TB is resistant to rifampicin, there is a good chance it may also be resistant to some

of the other drugs. In other words: multidrugresistant (MDR) TB.

In countries with widespread MDR TB, such as South Africa or throughout Eastern Europe, this ability is valuable. However, in countries where drug resistance remains limited, this resistance test has a low predictive value, meaning that any samples that look like they might be resistant to rifampicin need to be sent to a reference lab to be confirmed. In Zambia, there are only three such labs - and few clinics carry alternative drug regimens.

This is the catch with the use of sophisticated technology in such low-resource settings, says

\section{"If they don't} invest in TB, then all the money they have put into investing in HIV treatment will just be wiped away." Muyoyeta. "It's a problem because you're identifying these patients and then you can't do anything." This would be especially problematic in more remote parts of the country, which are many days travel from a reference laboratory.

Take the case of Tobias Hamooya, back at the Macha Mission Hospital in southern Zambia, where it is more than a day's walk to the nearest small town. This is the second time Hamooya has been diagnosed with TB; the first time he stopped taking the drugs when he started to feel better. Consequently, it is likely that his infection has developed some resistance to first-line drugs, but being so far from a reference laboratory, this would take many weeks to confirm, even if it was picked up by GeneXpert.

The jury then is still out on whether GeneXpert will live up to its expectations in Zambia. Muyoyeta and her team will publish the findings of the ZAMBART studies in the next few months. She acknowledges the amount of work required before GeneExpert can fulfil its potential. "We refer to GeneXpert as a newborn baby - it needs a lot of attention and is very demanding."

And as Carol Nyirenda, a Zambian activist who sits on the Stop TB Partnership's New Diagnostics Working Group, points out, failing to tackle TB undermines the tremendous progress that has been made to keep people living with HIV alive for longer. "In the earlier days if you were told you have HIV it was like a death sentence," says Nyirenda, who is living with HIV and is a TB survivor. "I've been on medication [ARVs] for close to ten years. TB could come and take me away within a week or two. If they don't invest in TB, then all the money they have put into investing in HIV treatment will just be wiped away."

Catherine de Lange is Naturejobs editor and travelled to Zambia as a new media journalism fellow with the International Reporting Project.

1. Padmapriyadarsini, C., Narendran, G. \& Swaminathan, S. Indian J. Med. Res. 134, 850-865 (2011).

2. World Health Organization. WHO Monitoring of Xpert MTB/RIF Roll-out (World Health Organization, 2013).

3. Lawn, S. D. et al. Lancet 12, 201-209 (2012). 